\title{
Influencia del Internet en el Rendimiento Académico de los Estudiantes de Educación Básica Regular
}

\author{
Yuri Misael Diaz Meza \\ Universidad Nacional Daniel Alcides Carrión \\ ORCID: https://orcid.org/0000-0003-4490-2033 \\ email: ydiazm@undac.edu.pe \\ Teófilo Félix Valentín Melgarejo \\ Universidad Nacional Daniel Alcides Carrión \\ ORCID: https://orcid.org/0000-0002-4063-5516 \\ email: tvalentinm@undac.edu.pe \\ Jose Rovino Alvarez Lopez \\ Universidad Nacional Daniel Alcides Carrión \\ ORCID: https://orcid.org/0000-0002-0019-3872 \\ email: jalvarez@undac.edu.pe \\ Juan Guillermo Ortiz Recinas \\ Unidad de Gestión Educativa Local - Pasco \\ ORCID: https://orcid.org/0000-0003-2742-1916 \\ email: jgortizr60@gmail.com
}

\section{RESUMEN}

La investigación influencia del internet en el rendimiento académico de los estudiantes del nivel de Educación Secundaria de Educación Básica Regular de Cerro de Pasco, provincia y región Pasco - Perú, tuvo como objetivo principal determinar la influencia del uso de internet en el rendimiento académico, asimismo; describir como favorece en los aprendizajes de los estudiantes, y explicar en qué medida el uso del internet mejora el rendimiento académico de los estudiantes. El tipo de investigación fue básica de nivel descriptivo - explicativo, se utilizó el método científico, además el hipotético - deductivo, en una muestra de 92 estudiantes de tipo probabilístico estratificada. Se hizo uso la encuesta en la aplicación del cuestionario de 26 preguntas, obteniendo como resultado según el estadígrafo de Correlación de Pearson 0,639, nivel de correlación alta, lo que permitió determinar que el uso de internet influye en el rendimiento académico de manera significativa.

Palabras clave: internet, rendimiento académico, enseñanza, aprendizaje. 


\title{
Influence of the Internet on the Academic performance of regular Basic Education Students
}

\begin{abstract}
The research influence of the internet on the academic performance of students at the level of Secondary Education of Regular Basic Education of Cerro de Pasco, Pasco province and region - Peru, had as main objective to determine the influence of internet use on academic performance, as well; describe how it favors student learning, and explain to what extent the use of the internet improves students' academic performance. The type of research was basic descriptive - explanatory level, the scientific method was used, in addition the hypothetical - deductive, in a sample of 92 students of stratified probabilistic type. The survey was used in the application of the 26-question questionnaire, obtaining as a result according to the Pearson Correlation statistic of 0.639, high correlation level, which allowed determining that the use of the Internet influences academic performance in a significant way.
\end{abstract}

Keywords: internet, academic performance, teaching, learning.

Artículo recibido: 05 de Abril 2021 Aceptado para publicación: 28 de Mayo 2021 Correspondencia: ydiazm@undac.edu.pe Conflictos de Interés: Ninguna que declarar 


\section{INTRODUCCIÓN}

En una sociedad caracterizada por el desarrollo de la información y la comunicación a través de las nuevas tecnologías, el internet es sin duda el fenómeno tecnológico de mayor importancia e incidencia en la actualidad, por ofrecer a la sociedad distintas formas para acceder a la información y posibilitar la comunicación sincrónica y asincrónica.

El desarrollo tecnológico permite hoy en día acceder a grandes recursos de información, procesarlos y transformarlos, a través del uso masivo de una red global interconectada a otras redes, las cuales permiten la comunicación y el intercambio de información a través de una tecnología avanzada con el fin de satisfacer necesidades de diferente índole (Acero, et al. 2008, p. 16), la estructura del desarrollo cognitivo de las personas, viene cambiando radicalmente por el impacto de la internet y otros medios que a través de los cuales las personas se comunican y aprenden, por los mecanismos con que acceden a los servicios que ofrecen al campo de la educación, por esta razón; es necesario concienciar a la población sobre el uso de la internet y sus implicancias. No cabe duda de que internet nos ha hecho la vida más fácil, especialmente en el campo de la educación, pero todavía no hemos realizado los avances que cabría esperar en el siglo XXI (Demdam, 2016). Cada vez es más amplio el uso del internet, proporciona un cúmulo de información en su mayor parte gratuita y de libre acceso, beneficiando a todos los actores educativos, siempre en cuando le den un uso apropiado que en los distintos ámbitos.

Al respecto Pardilla, 2018, citado en Cárdenas, 2019, menciona:

Es preciso reconocer que el mundo evoluciona cada día y con cada cambio surgen nuevas maneras de realizar las cosas, y la educación no es ajena a dichos cambios, por ello es necesario que se incorporen recursos que permitan un aprendizaje rápido y eficaz en los estudiantes. Entre las herramientas que benefician a la educación se encuentra el internet, el cual, unido a los dispositivos electrónicos permite trascender barreras, logrando que la tecnología mejore los conocimientos y actividades de los educadores y de los educandos. (p. 12)

La situación educativa de un país, donde el uso internet se ha convertido en una de las herramientas más utilizadas en el proceso e intercambio de experiencias de aprendizajes, el rol del docente y del estudiante ha cambiado, actualmente el docente desempeña nuevas funciones, desde buscar información, diseño de medios, materiales y recursos, teniendo 
en cuenta las características de los estudiantes; que al hacer el uso adecuado de este medio puede elevar el rendimiento académico y mejorar las calificaciones en las distintas áreas curriculares, de esta manera concretando sus conocimientos y capacidades.

El sistema educativo peruano, se ha visto vulnerado por el uso indiscriminado del internet en los jóvenes estudiantes, que en muchos de los casos lo hacen uso por diversión, por pasar el tiempo o por desconocimiento de su importancia en su formación pedagógico y académica que le ayudarían obtener un rendimiento académico satisfactorio, asimismo; la educación y los estudiantes del contexto educativo de Cerro de Pasco no es ajeno a esta realidad, por lo que, no vienen aprovechando el internet de manera apropiada con fines de aprendizajes y rendimientos académicos, lo cual es materia de análisis para llegar a un propuestas, resultados y conclusiones de la investigación.

En la investigación, uso de internet como herramienta pedagógica para facilitar el aprendizaje elaborativo y profundo; parte de sus conclusiones hace mención que los estudiantes mejoraron significativamente el rendimiento, si se comparan los resultados del test diagnóstico con el certamen final (Salinas, 2004, p. 55), asimismo; en otra investigación, Internet y rendimiento académico caso: Estudiantes de la Universidad Católica sedes Sapientiae Tarma; concluye que, el resultado de la relación entre el internet y rendimiento académico, nos da a entender que existe a mayor uso aumentara el rendimiento académico, por tanto se debe seguir investigando el presente estudio (Moreno, 2018, p. 81). Lo que permite aseverar que el uso pertinente del internet en el aspecto educativo promueve el rendimiento académico.

La investigación es importante porque permitirá determinar, una situación que nos concierne a todos los actores de la comunidad educativa frente a los efectos del internet en el rendimiento académico; al margen de que también nos brinda un mundo nuevo de interrelación, búsqueda de información y más servicios que son necesarios y útiles si lo sabemos aprovechar bien los estudiantes del nivel de Educación Secundaria de Educación Básica Regular de Cerro de Pasco, quienes son los que más uso dan a esta herramienta y recursos.

Por otra parte, conociendo la influencia del internet en el rendimiento académico de los estudiantes, se puede proponer nuevas formas y estrategias de enseñanza - aprendizaje, y un adecuado uso de esta herramienta y de los medios con fines educativos, que ayudaría 
no solo a tener un rendimiento adecuado en las distintas asignaturas, sino beneficiaria en el logro de metas y en la calidad de vida de los estudiantes.

$\mathrm{Al}$ respecto, es necesario tener en consideración los fundamentos conceptuales y teórico de internet y su influencia en el rendimiento académico, y la relaciona con los procesos de logro y procesos pedagógicos en los aprendizajes.

La naturaleza participativa y comunitaria de muchas de las aplicaciones y actividades sociales de internet está en consonancia con las características fundamentales del aprendizaje humano, en concreto, crear, compartir, colaborar y analizar (Selwyn, 2014, p. 197), y con la aparición de internet ha provocado un fuerte impacto en el ámbito educativo ocasionando cambios significativos en los métodos de enseñanza vigentes hasta hoy. Internet puede ser aplicado sirviendo de apoyo a la enseñanza tradicional, alternando ambos métodos, de forma que se complementen o también sustituyendo las clases educativas que conocemos. (Bermudez, 2008, citado en Paredes \& Rodríguez, 2008, p. 8). Por lo que, internet favorece la apertura y acceso al conocimiento, la capacidad de hacer, aportar o rectificar postulados y propuestas, teniendo en cuenta la cantidad de información que puedas encontrar en el internet y que solo sería necesario comprender los conceptos sobre la dinámica de los procesos de la información y que permita utilizar los métodos pedagógicos con los cuales el estudiante puede aprender mejor.

Asimismo, con respecto a rendimiento académico es complejo desde su conceptualización, debido a que en ocasiones se lo nombra, también, aptitud escolar y desempeño académico (Ortega \& González, 2016, p. 19).

Esta pueda ser definido como la relación entre lo obtenido y el esfuerzo empleado para obtenerlo, que refleja el resultado de las diferentes y complejas etapas del proceso educativo y al mismo tiempo, una de las metas hacia las que convergen todos los esfuerzos y todas las iniciativas de las autoridades educacionales, maestros, padres de familia y alumnos (Segura, 2010, p. 38). Además; rendimiento académico es el nivel de logro que puede alcanzar un estudiante en el ambiente académico en general o en una asignatura en particular. El mismo puede medirse con evaluaciones pedagógicas, entendidas éstas como el conjunto de procedimientos que se planean y aplican dentro del proceso educativo, con el fin de obtener la información necesaria para valorar el logro, por parte de los estudiantes, de los propósitos establecidos para dicho proceso (Sacachipana, 2017, p. 51), que tiene como propósito alcanzar una meta educativa, un 
aprendizaje. Esto implica la trasformación de un estado determinado en un estado nuevo; el rendimiento varía de acuerdo con las circunstancias, condiciones orgánicas y ambientales que determinan las aptitudes y experiencias (Champa, 2017. p. 46.)

El rendimiento académico incluye el del profesorado, así como el rendimiento de los recursos didácticos y aun del sistema educativo (Acero, et al. 2008, p. 14), en concordancia a los conocimientos escolares adquiridos por el estudiante, las capacidades intelectuales y el aprovechamiento que de ellas hace (Portellano, 1989; citado en Acero, et al. 2008, p. 15).

$\mathrm{Al}$ respecto, el rendimiento académico es el resultado de un nivel de exigencia para la valoración del aprendizaje logrado, y esto se observa en las calificaciones obtenidas, lo cual permite una mayor seguridad de que el objetivo central de la educación y del aprendizaje del estudiante, se haya alcanzado.

Con respecto a la influencia del internet en el rendimiento académico, el internet genera un interés en los estudiantes al momento de recibir sus clases y de reforzar en sus hogares dichos conocimientos adquiridos (Cárdenas, 2019, p. 12); es una modalidad de enseñanza online, es exitoso y no requiere un sobreesfuerzo o trabajo extraordinario en el profesorado ni en el alumnado (Area-Moreira, et al. 2020, p. 49); puesto que, el aprendizaje interactivo ha demostrado ser una herramienta eficaz en la mejora del rendimiento académico de los estudiantes de educación presencial y virtual (Bernard et al., 2009; Castaño-Muñoz, 2011; Castaño-Muñoz, Duart \& Sancho-Vinuesa, 2014, citado en Castaño, et al. 2015, p. 25).

El uso del internet por parte de los estudiantes incide en su rendimiento académico, el cual facilita la enseñanza aprendizaje y además proporciona diferentes herramientas educativas tanto a estudiantes como a docentes (Veliz, 2016, citado en Ccahuantico, 2018, p. 13), y sus beneficios, hace que los alumnos, usan como un herramienta y medio informático, y lograr nuevos revolucionarios conocimientos, esta herramienta poderosa fuente de consulta, académico ayuda a los procedimientos de aprendizaje, siendo utilizado en una educación virtual a fin de incrementar el rendimiento académico (Segura, 2011, citado en Moreno, 2018, p. 28).

Por lo que, realizar los procesos pedagógicos con el apoyo del internet permite lograr el rendimiento académico, hace que el docente promueva diversos recursos y habilidades; 
y los estudiantes en desarrollar más las capacidades y competencias en comprender adecuadamente sus desempeños y evidencias de aprendizaje.

Ahora bien, el proceso del aprendizaje no puede darse al margen del desarrollo tecnológico y avance del internet, este recurso plantea nuevos retos en el sistema educativo y en el proceso de enseñanza - aprendizaje, lo que nos lleva a contextualizar el estudio sobre la influencia del internet en el rendimiento académico, en el ámbito peruano. El rendimiento académico como el nivel de logro que puede alcanzar un estudiante en el ambiente escolar en general o en una asignatura en particular. El mismo puede medirse con evaluaciones pedagógicas, entendidas éstas como "el conjunto de procedimientos que se planean y aplican dentro del proceso educativo, con el fin de obtener la información necesaria para valorar el logro, por parte de los estudiantes, de los propósitos establecidos para dicho proceso" (Vega, 1998, citado en Segura, 2010. p. 20).

Para verificar si un estudiante logro un rendimiento académico favorable o desfavorable, es necesario que tener en consideración las escalas de medición o calificación, que permita verificar el logro de aprendizaje, el cual puede variar en función a los logros obtenidos por los estudiantes en una institución educativa.

Según, MINEDU (2016), establece conclusiones descriptivas del nivel de aprendizaje alcanzado por el estudiante, en función de la evidencia recogida en el período a evaluar; así como se asocian estas conclusiones con la escala de calificación (AD, A, B o C) para obtener un calificativo. (p. 105), es la siguiente:

\begin{tabular}{|c|c|l|}
\hline $\begin{array}{c}\text { Calificación } \\
\text { vigesimal }\end{array}$ & $\begin{array}{l}\text { Calificación } \\
\text { literal }\end{array}$ & \multicolumn{1}{|c|}{ Descripción del aprendizaje logrado } \\
\hline$[18-20]$ & AD & $\begin{array}{l}\text { Logro destacado } \\
\text { Cuando el estudiante evidencia un nivel superior a lo esperado } \\
\text { respecto a la competencia. Esto quiere decir que demuestra } \\
\text { aprendizajes que van más allá del nivel esperado. }\end{array}$ \\
\hline$[14-17]$ & A & $\begin{array}{l}\text { Logro esperado } \\
\text { Cuando el estudiante evidencia el nivel esperado respecto a la } \\
\text { competencia, demostrando manejo satisfactorio en todas las tareas } \\
\text { propuestas y en el tiempo programado. }\end{array}$ \\
\hline$[11-13]$ & $\mathrm{B}$ & $\begin{array}{l}\text { En proceso } \\
\text { Cuando el estudiante está próximo o cerca al nivel esperado respecto } \\
\text { a la competencia, para lo cual requiere acompañamiento durante un } \\
\text { tiempo razonable para lograrlo. }\end{array}$ \\
\hline$[0-10]$ & $\mathrm{C}$ & $\begin{array}{l}\text { En inicio } \\
\text { Cuando el estudiante muestra un progreso mínimo en una } \\
\text { competencia de acuerdo al nivel esperado. Evidencia con frecuencia } \\
\text { dificultades en el desarrollo de las tareas, por lo que necesita mayor } \\
\text { tiempo de acompañamiento e intervención del docente. }\end{array}$ \\
\hline
\end{tabular}

Fuente: Adaptado por los investigadores 
En este proceso es necesario la orientación del aprendizaje, guiar el proceso de aprender a aprender a fin de que la mayor parte de la información que se recibe pueda llegar a transformarse en conocimiento y sabiduría (Albero, 2001, p. 15), por lo que, es pertinente desarrollar las habilidades, capacidades y competencias para aprender de distintas formas en una sesión de aprendizaje y lograr desempeños favorables y duraderos y que esto sea evidenciado en productos o resultados de aprendizajes.

Al estructurar la sesión de aprendizaje se deben tener en cuenta tres momentos. Sin embargo, los momentos no deben plantearse de manera aislada, sino a partir de una secuencia didáctica (Zavala \& Arnao, 2008, citado en MINEDU, 2019, p. 56). Estos momentos se especifica en el siguiente gráfico:

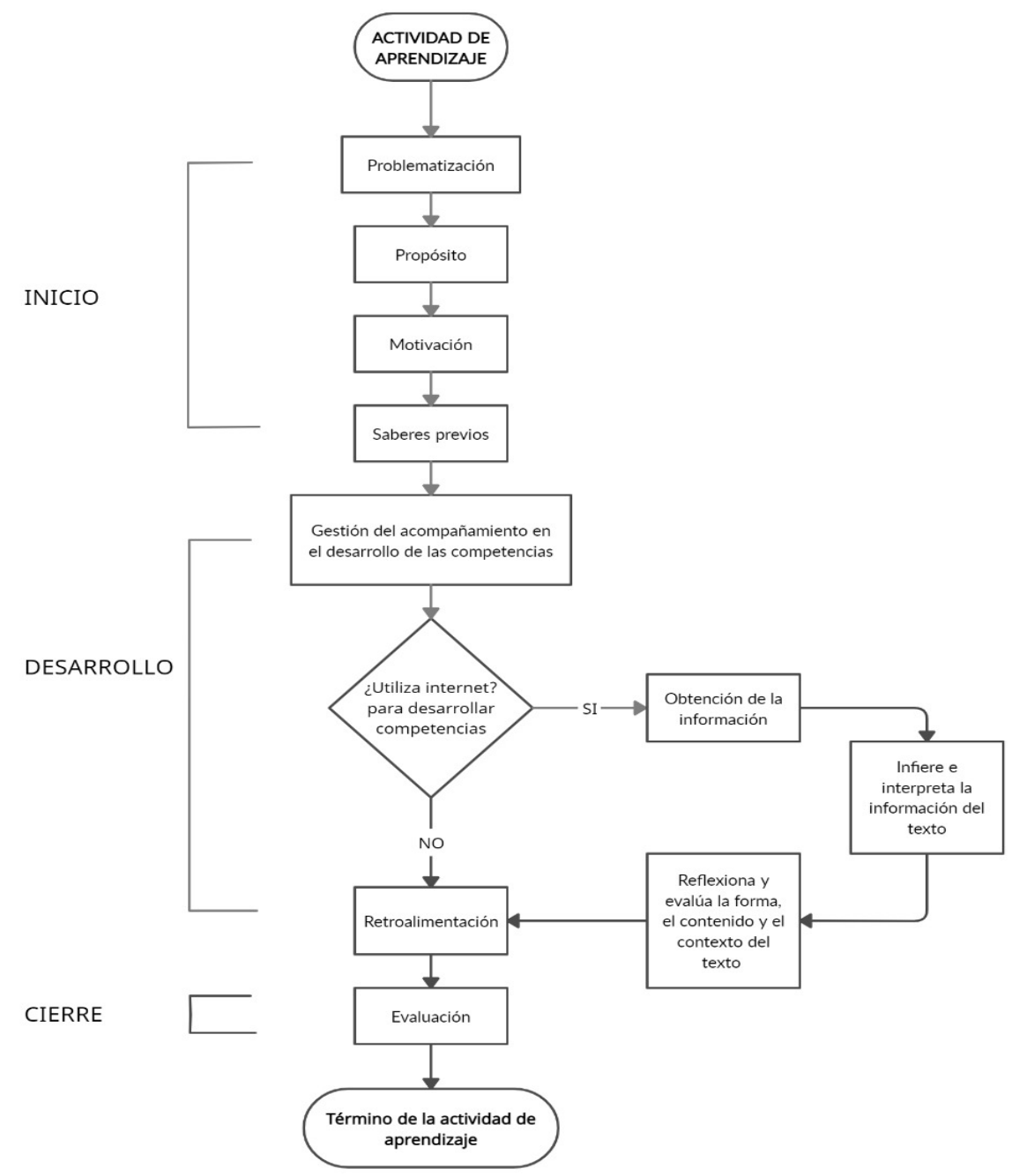

Fuente: Propuesta elaborado por los investigadores.

La actividad de aprendizaje inicia con el planteamiento de una problemática al estudiante, luego de ello se entrega el propósito de la actividad lo cual está relacionado con la motivación que se generará al estudiante; se recibe los saberes previos y dentro de la 
gestión del acompañamiento de las competencias se desarrolla las capacidades según la competencia elegida. En este caso la competencia lee diversos tipos de textos escritos en lengua materna, implica la combinación de las siguientes capacidades: obtiene información del texto escrito, infiere e interpreta información del texto, y reflexiona y evalúa la forma, el contenido y contexto del texto (MINEDU, 2016, p. 99); concluido los procesos anteriores se prosigue con la retroalimentación realizada por el docente; finalmente se da la evaluación del proceso con la colaboración y participación de los estudiantes, para evidenciar el rendimiento académico a través de sus desempeños.

En las actividades propuestas, se debe observar el rol del docente como mediador del aprendizaje y el del estudiante como protagonista-constructor del aprendizaje (MINEDU, 2019, p. 56).

El tutor o gestor del acompañamiento en el desarrollo de competencias, actúa guiando a los estudiantes en el proceso de aprendizaje, identifica tanto los niveles de conocimientos adquiridos, como los vacíos que ellos poseen, para tomar decisiones oportunas y logar un buen rendimiento académico.

El estudio tuvo como objetivo principal determinar la influencia del uso de internet en el rendimiento académico de los estudiantes del nivel de Educación Secundaria de Educación Básica Regular de Cerro de Pasco, provincia y región Pasco - Perú, asimismo; describir como favorece este en los aprendizajes de los estudiantes, y explicar en qué medida el uso del internet mejora el rendimiento académico de los estudiantes.

\section{METODOLÓGIA}

La investigación fue de tipo básica o teórica, orientada a la búsqueda de nuevos conocimientos sin una finalidad práctica específica e inmediata. Busca principios y leyes científicas, pudiendo organizar una teoría científica (Sánchez, et al. 2018, p. 79), para este caso se analiza la influencia del uso de Internet en el rendimiento académico de los estudiantes. El nivel de investigación fue descriptivo - explicativo, porque tuvo como propósito describir y explicar el estado actual y su la influencia del internet en el rendimiento académico de los estudiantes. El diseño para este estudio fue transeccional correlacional/causal debido a que los datos obtenidos fueron recogidos en un solo momento y en un tiempo único. Se utilizó el método hipotético - deductivo, de conocimiento relativo al método científico por el cual se hace uso de procedimientos lógicos deductivos, partiendo de un supuesto o planteamiento a priori que hay que 
demostrar (Sánchez, et al. 2018, p. 91), en la que se realizó un procedimiento a lo largo de la investigación.

La población estuvo conformada por 120 estudiantes de tres instituciones educativas del cuarto grado del nivel de Educación Secundaria de Educación Básica Regular de Cerro de Pasco, provincia y región Pasco - Perú, en el año académico 2019; de lo cual se obtuvo una muestra representativa de 92 estudiantes, de tipo probabilística estratificada.

Para la recolección de datos se utilizó la técnica de la observación y la encuesta, asimismo; el instrumento utilizado fue el cuestionario con 26 preguntas sobre las variables de investigación, para lo cual; se realizó el proceso de confiabilidad a un 95\% a través del método de Alfa de Cronbach, habiendo obtenido un coeficiente de 0,887 como resultado, que especifica una confiabilidad aceptable, además; se realizó la prueba de validación a través del juicio de expertos y la prueba piloto del respectivo instrumento. Luego se procedió a la aplicación del instrumentos y procesamiento de los datos, para lo cual se hizo uso de las herramientas informáticos como el Microsoft Excel versión 2016 y el programa SPSS versión 21.

\section{RESULTADOS Y DISCUSIÓN}

Los resultados de los datos obtenidos de las variables de estudio se centralizaron en frecuencias y porcentajes, se establecieron medidas de tendencia central (media) y medidas de variabilidad (desviación estándar); estos resultados se analizaron por ítems, de manera cuantitativamente y cualitativamente, haciendo uso de la estadística descriptiva e inferencial, en el que; se hizo uso del estadígrafo de prueba para las variables a través de la correlación de Pearson, donde se obtuvo como coeficiente de contingencia el siguiente resultado.

\begin{tabular}{|c|c|c|}
\hline $\begin{array}{c}\text { Variable } \\
\text { independiente }\end{array}$ & $\begin{array}{c}\text { Variable } \\
\text { dependiente }\end{array}$ & $\begin{array}{c}\text { Coeficiente de } \\
\text { contingencia }\end{array}$ \\
\hline Internet & $\begin{array}{c}\text { Rendimiento } \\
\text { académico }\end{array}$ & 0,639 \\
\hline
\end{tabular}

- Correlación de Pearson Sig (Bilateral)

- $\mathrm{C}=$ Correlación de Pearson =0,639

El coeficiente de correlación de Pearson, toma valores comprendidos entre cero y uno, cuando está próximo a cero, indica una asociación nula, baja o muy débil (no significativa) entre las variables involucradas, y cuando está próximo a uno, indica una 
asociación alta, fuerte, o casi perfecta (significativa), dependiendo de la cercanía al número uno.

Puesto que la Correlación de Pearson es igual a 0,639 y se aproxima a 1, asimismo; es $<1$. Por lo que; el uso del internet influye significativamente en el rendimiento académico de los estudiantes del nivel de Educación Secundaria de Educación Básica Regular de Cerro de Pasco, provincia y región Pasco - Perú. Sobre la base de los resultados analizados desde el ítem 01 hasta el ítem 13 con respecto a la variable independiente sobre la influencia en el uso de internet, se logró obtener de la muestra definida una Media de 46.86, de igual manera la Mediana fue 48.00, de igual manera la Desviación típica es de 4.56, como también se logró obtener la Varianza de 20.80, los cuales estos datos permitieron validar la información de nuestra variable independiente y considerando que esta es determinante en la presente investigación. Por la otra parte, considerando la variable dependiente, en nivel de rendimiento académico teniendo la estadística descriptiva y los datos obtenidos desde el ítem 14 al 26 y la relación que existe entre la variable independiente y la dependiente, se obtuvo los siguientes resultados: una Media de 57.23, de igual manera la Mediana es de 59.00, la Desviación típica es de 5.76 y la Varianza es de 33.17.

Con respecto a los datos descritos de acuerdo a las variables; el uso de internet influye significativamente en el rendimiento académico de los estudiantes del nivel de Educación Secundaria de Educación Básica Regular de Cerro de Pasco, provincia y región Pasco Perú.

\section{CONCLUSIONES}

1. La influencia del uso de internet y el rendimiento académico del nivel de Educación Secundaria de Educación Básica Regular de Cerro de Pasco, provincia y región Pasco - Perú, es significativa.

2. Las técnicas y el instrumento utilizado en la presente investigación, permitieron garantizar el recojo y procesamiento de los datos, por su validez y confiabilidad de acuerdo a los análisis estadísticos practicados, tal como se demuestra en los resultados.

3. Los resultados obtenidos corroboran las conclusiones y resultados obtenidos en otras investigaciones, en la que el uso del internet por parte de los estudiantes mejoraron significativamente el rendimiento, si se comparan los resultados del test diagnóstico 
con el certamen final (Salinas, 2004, p. 55), y el resultado de la relación entre el internet y rendimiento académico, nos da a entender que existe a mayor uso aumentara el rendimiento académico, por tanto se debe seguir investigando el presente estudio (Moreno, 2018, p. 81).

4. En términos generales, los resultados obtenidos permiten describir y explicar que el uso del internet influye significativamente en el rendimiento académico de los estudiantes del nivel de Educación Secundaria de Educación Básica Regular de Cerro de Pasco, provincia y región Pasco - Perú.

\section{REFERENCIAS}

Acero, G., Talman, A., Vergara, A., Moreno, J. \& Jiménez, H. (2008). Relación entre hábitos de salud, consumo de internet y rendimiento académico en adolescentes de un colegio de la ciudad de Bogotá. Psicogente, 11 (19), 9-23.

Albero, M. (2001). Internet, escuela y vida cotidiana en la infancia. Telos 3 (1), 9-20. https://dialnet.unirioja.es/servlet/articulo?codigo $=6436391$

Area-Moreira1, M., bethencourt-Aguilar, A. \& Martín-gómez, S. (2020). De la enseñanza semipresencial a la enseñanza online en tiempos de Covid19. Visiones del alumnado. Campus Virtuales, 9(2), 35-50.

Cárdenas, J. (2019). El uso de internet y el rendimiento académico de los estudiantes del primer y segundo año de secundaria de la Institución Educativa Particular "Bertolt Brecht” Cercado de Lima 2015 [Tesis de título profesional, Universidad Cesar vallejo]. https://repositorio.ucv.edu.pe/handle/20.500.12692/40161

Castaño, J., Duart, J. M. \& Sancho-Vinuesa, T. (2015). Determinantes del uso de Internet para el aprendizaje interactivo: un estudio exploratorio. New Approaches in Educational Research 4(1), 25-34.

Ccahuantico, E. (2018). Uso del internet y el rendimiento académico de los estudiantes del segundo grado de secundaria de la Institución Educativa Cap. Alipio Ponce Vásquez de Puerto Maldonado - 2018 [Tesis de título profesional. Universidad Nacional Amazónica de Madre de Dios]. http://repositorio.unamad.edu.pe/handle/UNAMAD/423

Champa, R. R. (2017). El uso de internet y su relación con el rendimiento escolar en los estudiantes del V Ciclo de Educación Primaria de la I.E. Experimental de 
Aplicación de la UNE [Tesis de título profesional, Universidad Nacional de Educación]. https://repositorio.une.edu.pe/handle/UNE/1191

Demdam, M. (2016). Internet desempeña un papel importante en el sistema educativo. https://www.bbvaopenmind.com/tecnologia/mundo-digital/internet-desempenaun-papel-importante-en-el-sistema-educativo/

Hernández, R. Fernández, C., y Baptista, M. (2014). Metodología de la Investigación. McGraw-Hill

MINEDU (2019). Planificación, mediación y evaluación de los aprendizajes en la Educación Secundaria. Primera Edición. Ministerio de Educación del Perú.

MINEDU (2016). Currículo Nacional de la Educación Básica. Ministerio de Educación del Perú.

MINEDU (2016). Programa Curricular de Educación Secundaria. Ministerio de Educación del Perú.

Moreno, J. (2018). Internet y rendimiento académico caso: Estudiantes de la Universidad Católica sedes Sapientiae Tarma [Tesis de Maestría, Universidad Nacional del Centro del Perú]. http://repositorio.uncp.edu.pe/bitstream/handle/UNCP/4806/Moreno\%20Palomi no.pdf

Ortega, J. \& González, D. (2016). El ciberacoso y su relación con el rendimiento académico. Innovación Educativa, $16 \quad$ (71), $\quad$ 17-38. http://www.scielo.org.mx/pdf/ie/v16n71/1665-2673-ie-16-71-00017.pdf

Paredes, C. L. \& Rodríguez, S. Y. (2008). Influencia del uso de internet en el nivel de rendimiento académico del estudiante de la facultad de enfermería [Tesis para de título, Universidad Nacional de Trujillo]. http://dspace.unitru.edu.pe/handle/UNITRU/5722

Sacachipana, M. (2017). Influencia del uso del internet en el rendimiento académico de los estudiantes de la institución educativa secundaria Carlos Oquendo de AMAT del distrito de Cиуосиуо - 2012 [Tesis de título profesional, Universidad Nacional del Altiplano]. http://repositorio.unap.edu.pe/handle/UNAP/3661

Salinas, H. (2004). Uso de internet como herramienta pedagógica para facilitar el aprendizaje elaborativo y profundo [Tesis de Magister, Universidad de Chile]. http://www.tesis.uchile.cl/tesis/uchile/2004/salinas_h/sources/salinas_h.pdf 
Sánchez, H. H., Reyes, C. \& Mejía, K. (2018). Manual de términos en investigación científica, tecnológica y humanística. Universidad Ricardo Palma.

Segura, F. (2010). El uso del internet en el desarrollo del rendimiento académico de los estudiantes de los séptimos años de educación básica de la escuela fiscal mixta “Luis Vivero" de la parroquia Totoras Cantón Ambato durante el periodo juniooctubre del 2010 [Trabajo de Titulación, Universidad Técnica de Ambato]. https://repositorio.uta.edu.ec/bitstream/123456789/588/1/CF-105.pdf

Selwyn, N. (2014). Internet y educación. https://www.bbvaopenmind.com/wpcontent/uploads/2014/03/BBVA-OpenMind-Internet-y-educacion-NeilSelwyn.pdf.pdf 